\title{
Application of Argon Plasma Jet for Methane Hydrate Decomposition by Radio Frequency Irradiation
}

\author{
Ismail Rahim ${ }^{\#}$, Shinfuku Nomura ${ }^{*}$, Shinobu Mukasa ${ }^{*}$, Hiromichi Toyota ${ }^{*}$, Muhammad Agung ${ }^{\#}$ and \\ Novriany Amaliyah ${ }^{* *}$ \\ ${ }^{\#}$ Department of Education for Automotive Engineering, Universitas Negeri Makassar, Makassar, 90222, Indonesia. \\ E-mail: ismail_rahim@unm.ac.id \\ *Graduate School of Science and Engineering, Ehime University, Matsuyama, 790-8577, Japan. \\ E-mail: nomura.shinfuku.mg@ehime-u.ac.jp \\ *** Mechanical Engineering Department, Hasanuddin University, Makassar, 92119, Indonesia.
}

\begin{abstract}
In this study, decomposition of methane hydrate using argon plasma jet investigated in the pressure range of $0.1 \mathrm{MPa}$ to 2.0MPa. The plasma generated under the high-pressure condition, which is difficult to achieve when using radio frequency (RF) plasma in the liquid method. By using emission spectrometer analysis, the excitation temperature is found to increase as the gas pressure increases, whereas, it decreases as the argon flow rate increases. During the process of plasma irradiation, the required essential reactions for methane hydrate decomposition, such as methane hydrate dissociation (MHD), steam methane reforming (SMR), and methane cracking reaction (MCR) were not completely satisfied due to an insignificant amount of methane. The gas chromatography analysis confirmed that the methane cracking reaction (MCR) was only occurred to generate hydrogen and the $\mathrm{C}_{(\mathrm{s})}$, due to the absence of $\mathrm{C}_{2} \mathrm{H}_{2}$ and $\mathrm{C}_{2} \mathrm{H}_{4}$ as the byproducts. In comparison with the other primary reactions of methane hydrate decomposition, steam methane reforming reaction became dominant in converting methane into hydrogen. Although the hydrogen production efficiency is less than that of radio frequency plasma in liquid, the reduction of $\mathrm{CO}_{2}$ by the thermal decomposition of Teflon from $\mathrm{CO}$ making it possible is considered as an advanced promising technique in the future.
\end{abstract}

Keywords — argon plasma jet; methane hydrate; hydrogen production; radio frequency; emission spectroscopy.

\section{INTRODUCTION}

Environmental severe problems in the world by the burning of fossil fuels and the increasing of greenhouse gases emission including carbon dioxide have paid our attention nowadays. The consideration has been focused on alternative energy sources such as solar energy, biomass, biodiesel [1], geothermal power, and tidal power. All of these natural energy resources are presumed to minimize our dependency on fossil fuel. Hence, due to its characteristic as a clean energy carrier and environmental green nature [2], hydrogen has been pointed out as a vital fuel to be used which could substitute the current combustion engines in which diesel and or gasoline used in common. However, for the reason that hydrogen cannot be considered as a renewable fuel, it has to be obtained from other primary hydrogen-enriched sources such as hydrocarbon or alcohol [3] and biomass and organic waste [4].

Methane hydrates have become one of the most attractive for hydrogen energy resources for the future due to its massive reserves in the world, which is possible twice the global amount of carbon of all other fossil fuels combined [2], [5]. They are crystalline solids formed by a compound of methane and water which stable at low temperature and high pressure which have an ice-like crystalline lattice of water molecules with methane molecules trapped inside. Huge resources of hydrates can be found on the ocean floors of continental shelves and in the permafrost region [2], [5]. Due to the solid form of methane hydrates, the recovery of methane involves dissociating or melting methane hydrates in situ by raising the temperature above that of hydrate formation or decreasing the pressure below that of hydrate equilibrium [2]. Therefore, conventional techniques for gas and oil recovery are not applicable.

The application of the in-liquid plasma method by radio frequency (RF) under atmospheric pressure has been successfully applied to produce $65 \%$ of hydrogen from cyclopentane (CP) [6], and also from waste oil and ndodecane with $70-80 \%$ of hydrogen [7], [8]. This method has also provided satisfactory results to produce hydrogen 
from the decomposition of methane hydrate [7], [11]. However, in the case of its application under severe environments such as high pressure and low temperature, it was found that it is relatively formidable to obtain a stable plasma irradiation. For the conventional in-liquid plasma method, the plasma can be generated as long as the applied electric field across the discharge gap is high enough to initiate a breakdown. However, under the condition of high pressure, the electric field that required to stimulate the discharge is fairly high [12]. Thus, a suitable method is required to produce hydrogen under critical conditions since methane hydrates are stable only at such conditions [9], [10].

The study of plasma jet has received much attention recently due to the versatility of its application. Some of the attention are synthesis of carbon nanotubes [13], microparticles/microsphere production [14], [15], surface modification[16], diamond deposition [17], and hydrogen production[18]. Methane Reformation using a gliding plasma jet reactor can produce hydrogen-rich gas with a $54 \% \mathrm{H}_{2}$ yield [19]. Hydrogen generation by the pulsed discharge of plasma jet can produce an optimum energy yield when using an argon carrier and pure methanol [18]. Likewise, discharge plasma in plasma jet method is extended beyond the plasma generation region into the surrounding ambiance by an electromagnetic field, convective gas flow, shockwave, or a gradient of a directionless physical quantity such as particle density, pressure, or temperature [20]. The combination of its ability to penetrate and propagate into narrow gap and flexible dielectrics makes this method easy to generate plasma with stable irradiation under the highpressure condition [21], [22].

In this study, the argon plasma jet adopted as the method for the decomposition process of methane hydrate ranging from 0.1 to $2.0 \mathrm{MPa}$ because this method is applicable under the condition of high pressure. Argon plasma jet is not confined by electrodes and can be adjusted to the small gap [22]. As well, there are yet no studies reported regarding of the characteristics of plasma under the high-pressure condition, also the decomposition process of methane hydrate by the argon plasma jet method. The analysis of emission spectrum of the plasma jet and the gas yield, as well as the efficiency of hydrogen production from the decomposition process, are investigated in this study. This is another step in the process with the ultimate goal of producing hydrogen from hydrate fields on the seabed and in permafrost regions using an in-liquid plasma method.

\section{MATERIAL AND METHOD}

\subsection{Formation of methane hydrate.}

Fig. 1 represent the apparatus for synthesizing methane hydrate which consists of a methane gas supply tube, cooling bath, stirring motor, magnetic stirrer, pressure gauge, thermocouple, and computer. About 90 grams of shaved ice and 10 grams of methane hydrate seed inserted into the cooling bath (60 mm inner diameter, $140 \mathrm{~mm}$ height, $400 \mathrm{ml}$ volume, and maximum working pressure of $15 \mathrm{MPa}$.), which has been previously washed by water. Pressurized methane gas then injected into the cooling bath, $30 \mathrm{~mm}$ above the bottom.

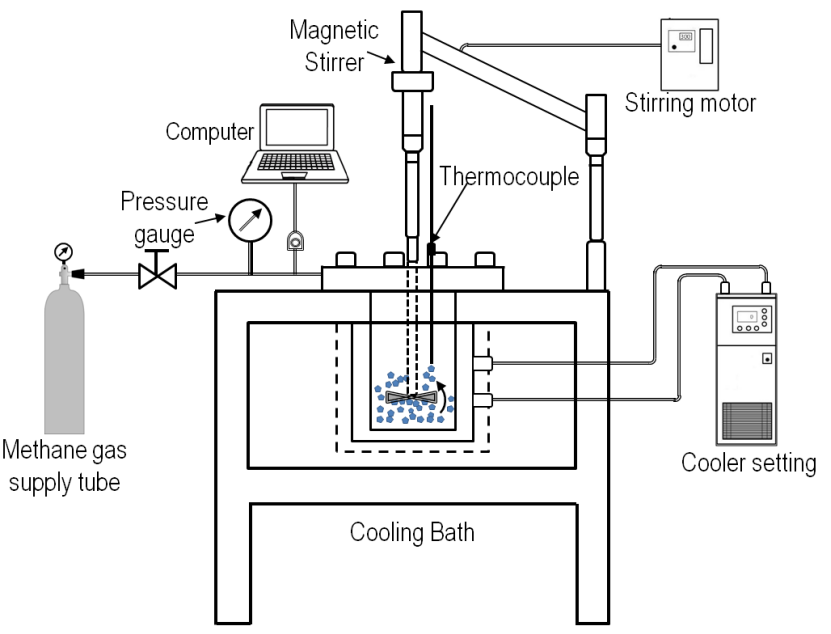

Fig.1 Apparatus for synthesizing methane hydrate

The temperature was maintained constant at $1^{\circ} \mathrm{C}$ using ethylene glycol, and a magnetic stirrer $(40 \mathrm{~mm})$ rotated at $500 \mathrm{rpm}$ to stir up the solution of methane gas and shaved ice. Methane gas pressurized to about 7.0MPa, and the temperature of methane hydrate formation monitored by a thermocouple located at the underneath of the cooling bath. Pressure and temperature throughout the process recorded every hour. The methane hydrate formation process completed after 70 hours, and then synthetic methane hydrate collected. The methane hydrate was stored in a refrigerator to prevent the dissociation of the methane hydrate as a further cooling process, [23].

\subsection{Argon plasma jet irradiation for methane hydrate decomposition.}

The experimental apparatus of the argon plasma jet shown in Fig.2. For maintaining the high-pressure level, a pressure control valve attached to a gas outlet which increased the pressure inside the reactor vessel to the target pressure. The reactor temperature kept at $0^{\circ} \mathrm{C}$ by a surrounding copper tubing coil refrigerated by circulating ethylene glycol.

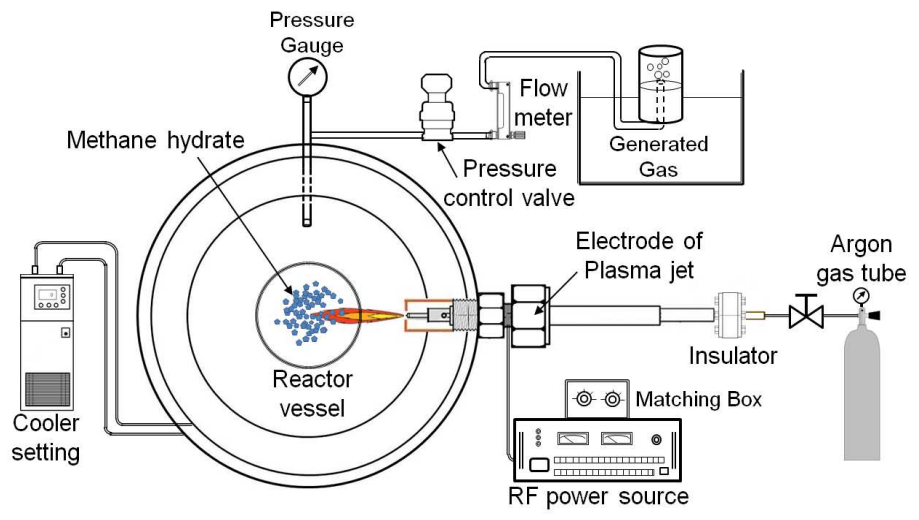

Fig.2 Experimental design of argon plasma jet irradiation for methane hydrate decomposition

Plasma irradiated by applying a $27.12 \mathrm{MHz}$ highfrequency power source (T161-5766LQ, Tramway) to an electrode unit. An insulator made of a heat-resistant polymer resin (PEEK, up to $500^{\circ} \mathrm{C}$ ) was attached to the apparatus to prevent energy loss and carbide generation. The electrode 
unit for the argon plasma jet applied in the experiment is shown in Fig.3. A $1.5 \mathrm{~mm}$ diameter electrode tip positioned on the axis of a $1.8 \mathrm{~mm}$ diameter stainless steel tube, where its surface insulated by another tube made of Teflon. A copper cylindrical tube installed as a counter electrode. Additionally, a hole for gas extraction drilled on the side wall of the stainless steel tube. A plasma jet irradiated from a $2.0 \mathrm{~mm}$ diameter hole drilled in the counter electrode through, which was the argon gas flowed from a stainless steel tube connected to the supply tube of argon. The Ar gas flow controlled at the desired rate of $200 \mathrm{~mL} / \mathrm{min}$ by a flow meter connected to the apparatus.

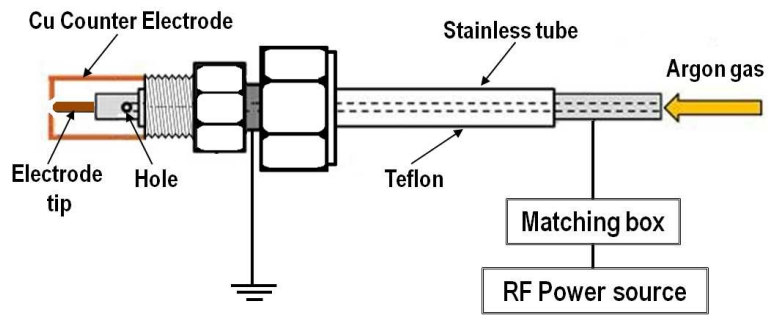

Fig.3 Electrode unit of the argon plasma jet

About $7 \mathrm{~g}$ of methane hydrate produced from the cooling bath inserted in the reactor vessel. The plasma jet input power was set to $250 \mathrm{~W}$ for a net power of $200 \mathrm{~W}$ after subtraction of the reflected power. Immediately after the plasma jet initiation, the pressure in the reactor vessel was adjusted to the target pressure of from $0.1 \mathrm{MPa}$ to $2.0 \mathrm{MPa}$ by adjusting the control valve. Plasma irradiation time recorded until the entire amount of methane hydrate dissolved, and the generated gases collected by the water displacement method. By this method, generated gas caught from the exhaust tube connected to the reaction vessel by displacing the gas above the liquid [6], [10]. Analysis of the gas content from the decomposition of methane hydrate was performed using a gas chromatograph (Shimadzu 8A), which argon as the carrier gas under a flow rate of $34 \mathrm{~mL} / \mathrm{min}$ and a head pressure of $600 \mathrm{kPa}$. The temperature for the column was 60 ${ }^{\circ} \mathrm{C}$, while the temperature of injector and detector were $160^{\circ} \mathrm{C}$

In this study, the gas yield defined by dividing the percentage of the ratio of the peak area of the gas content from gas chromatography analysis with the percentage of the total summation of gas content in the air with the amount of oxygen and nitrogen neglected. Also, during plasma irradiation, the emission spectroscopy measurement was conducted using a multichannel spectral analyzer (Hamamatsu Photonics-PMA 11 C7473-36).

\section{RESULTS AND DISCUSSION}

\section{A. Formation of methane hydrate}

There was a rapid formation of methane hydrate in the cooling bath at the beginning process after the stirrer was turned on. Then a concurrent pressure decrease was occurred to about $6 \mathrm{MPa}$ and a temperature increase of about $1{ }^{\circ} \mathrm{C}$ due to the exothermic reaction, as shown in Fig. 4. The basic formula of methane hydrate is $\mathrm{CH}_{4} \cdot n \mathrm{H}_{2} \mathrm{O}$, where $n$ is the stoichiometric number (hydrate number), which describes a variable number of water molecules in its lattice structure.
The formation of synthetic methane hydrate in the present study required around 70 hours at a pressure range of 6 to $7 \mathrm{MPa}$ and temperature range of 272 to $279 \mathrm{~K}$. By direct measurement, the hydrate number $(n)$ calculated from the amount of gaseous methane and water released during the hydrate decomposition at constant pressure.

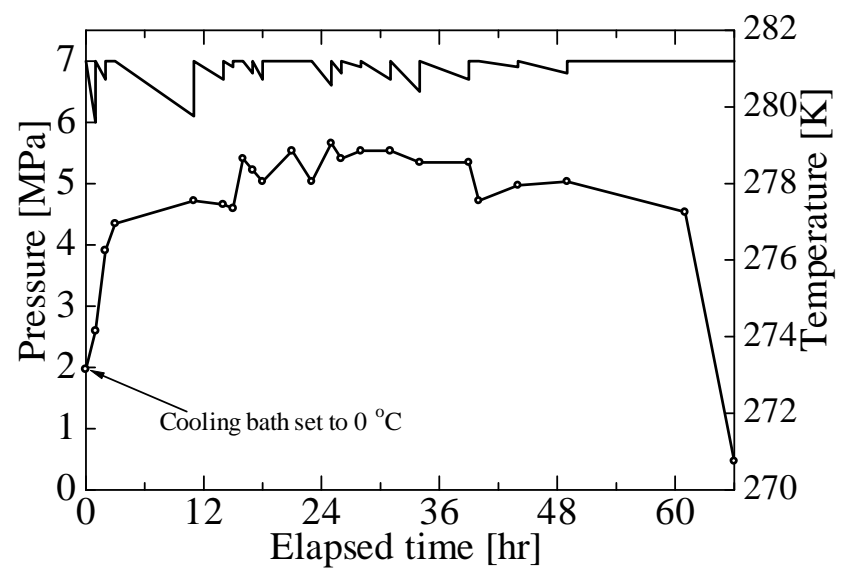

Fig. 4 Pressure and temperature of methane hydrate during the formation process.

A small amount of methane hydrate $( \pm 6$ grams) was taken from the 100 grams of synthetic methane hydrate produced. Values of 0.2896 of $\mathrm{mol} \mathrm{H}_{2} \mathrm{O}$ and 0.049107 of mol $\mathrm{CH}_{4}$ obtained after using the water displacement method and stoichiometric calculation. The $n$ value was then determined by the following equation:

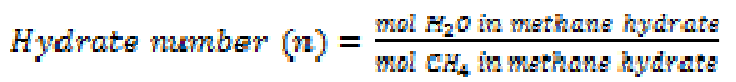

Hence, the hydrate number of synthetic methane hydrate in this experiment was determined to be 5.9, which can be written as $\mathrm{CH}_{4} \cdot 5.9 \mathrm{H}_{2} \mathrm{O}$ in the basic formula of methane hydrate. Along the natural methane hydrate equilibrium boundary for a stoichiometric number $\mathrm{n}=5.81$ to 6.10 [24]. The pressure ranges from 1.9 to $9.7 \mathrm{MPa}$, and the formation temperature ranges from 263 to $285 \mathrm{~K}$. Hence, the synthetic methane hydrate used in this experiment adequately represent the properties of actual methane hydrate in nature.

\section{B. Emission Spectroscopy of Argon Plasma Jet}

The variation in emission intensities of several ArI lines as a function of pressure shown in Fig. 5.

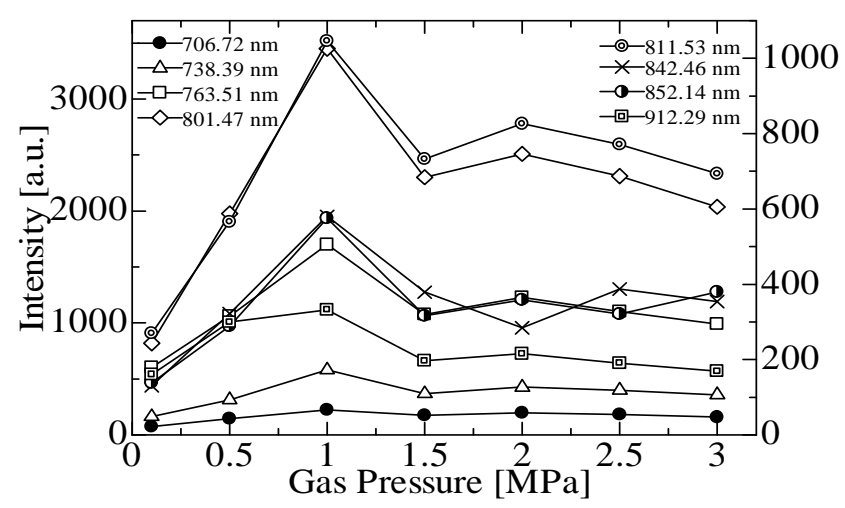

Fig.5 Optical Emission intensity of argon I line as a function of pressure 
Fundamentally, the emission intensity influenced by microscopic electric fields from the adjacent ions and electrons, which leads to a stark broadening of the spectral lines, which extended by increasing the pressure [25]. Based on previous research by Nomura et al., the gas temperature of plasma is known to be about $3500 \mathrm{~K}$ under atmospheric pressure [25]. In this experiment, the spectrum dominated by $\mathrm{Ar}$ and $\mathrm{Cu}$ emission lines. The emission line spectrum of the argon plasma jet at different pressures shown in Fig. 6.
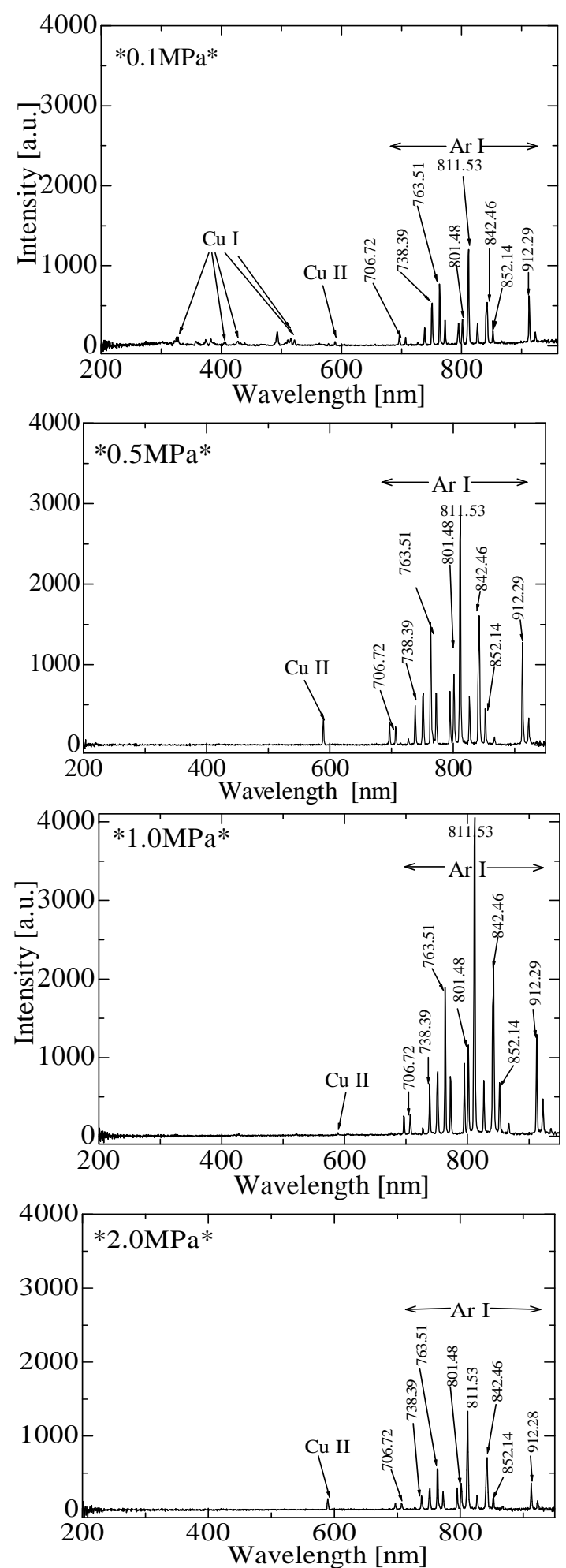

Fig.6 The emission line spectrum of the argon plasma jet at different pressures (flow rate $200 \mathrm{~mL} / \mathrm{min} \&$ pressure range of $0.1-2.0 \mathrm{MPa}$ )
The excitation temperature, $T_{\text {exc }}$ is a plasma parameter that characterizing a population of excited atomic levels [26]. This temperature evaluated from the inverse of the slope of a plot of the natural logarithm of $' 1_{i j} \lambda_{i j} /\left(\mathrm{g}_{i} \mathrm{~A}_{i j}\right)$ ' versus the transition of upper-level energy $\left(\mathrm{E}_{i}\right)$. Such a plot referred as a Boltzmann plot. The Boltzmann method employed under the assumption of local thermodynamic equilibrium (LTE) [27]-[31]. LTE plasma constraints that transitions and chemical reactions be dominated by collisions and not by the radiative processes, as well the local gradients of the plasma properties (temperature, density, thermal conductivity) are low enough to let a particle in the plasma reach an equilibrium [29].

The relationship between emission intensity of line $\left(\mathrm{I}_{i j}\right)$ and excitation temperature, $\mathrm{T}_{e x c}$ is shown by the following equation [26], [29]-[32]:

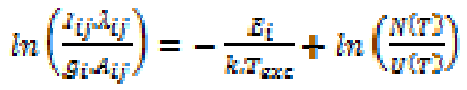

Where $\lambda_{i j}$ is the wavelength, $\mathrm{g}_{i}$ is the statistical weight of the upper level, $\mathrm{A}_{i j}$ is the transition probability, $\mathrm{E}_{i}$ is the upper-level energy, $k$ is the Boltzmann constant, $N(T)$ is the total number density of neutrals, and $U(T)$ is the partition function.

Fig. 7 constructs the Boltzmann plots from the net intensity of Argon I lines, the spectroscopic properties referred to in the NIST database [33]. Considering that $N(T) / U(T)$ is a standard constant for all lines under a specific temperature, $N(T) / U(T)$ neglected in the calculation. The spectral lines that have been used to estimate the excitation temperature are shown in Table 1.

TABLE I

THE SPECTRAL LiNES Of ARgON I USED For EsTIMATE THE EXCITATION TEMPERATURE

\begin{tabular}{|c|c|c|c|}
\hline \hline No. & $\begin{array}{c}\text { Substa } \\
\text { nce }\end{array}$ & Wavelength & Configuration of upper level \\
\hline 1 & Ar I & $706.7 \mathrm{~nm}$ & $3 \mathrm{~s}^{2} 3 \mathrm{p}^{4}\left[{ }^{3} \mathrm{P}\right] 5 \mathrm{p} \rightarrow 3 \mathrm{~s}^{2} 3 \mathrm{p}^{4}\left[{ }^{3} \mathrm{P}\right] 5 \mathrm{~d}$ \\
\hline 2 & Ar I & $738.4 \mathrm{~nm}$ & $3 \mathrm{~s}^{2} 3 \mathrm{p}^{5}\left[{ }^{2} \mathrm{P}^{\circ}{ }_{3 / 2}\right] 4 \mathrm{~s} \rightarrow 3 \mathrm{~s}^{2} 3 \mathrm{p}^{5}\left[{ }^{2} \mathrm{P}^{\circ}{ }_{1 / 2}\right] 4$ \\
\hline 3 & Ar I & $763.5 \mathrm{~nm}$ & $3 \mathrm{~s}^{2} 3 \mathrm{p}^{5}\left[{ }^{2} \mathrm{P}_{3 / 2}^{\circ}\right] 4 \mathrm{~s} \rightarrow 3 \mathrm{~s}^{2} 3 \mathrm{p}^{5}\left[{ }^{2} \mathrm{P}_{3 / 2}^{\circ}\right] 4$ \\
\hline 4 & Ar I & $801.5 \mathrm{~nm}$ & $3 \mathrm{~s}^{2} 3 \mathrm{p}^{5}\left[{ }^{2} \mathrm{P}^{\circ}{ }_{3 / 2}\right] 4 \mathrm{~s} \rightarrow 3 \mathrm{~s}^{2} 3 \mathrm{p}^{5}\left[{ }^{2} \mathrm{P}^{\circ}{ }_{3 / 2}\right]$ \\
\hline 5 & Ar I & $811.5 \mathrm{~nm}$ & $3 \mathrm{~s}^{2} 3 \mathrm{p}^{5}\left[{ }^{2} \mathrm{P}^{\circ}{ }_{3 / 2}\right] 4 \mathrm{~s} \rightarrow 3 \mathrm{~s}^{2} 3 \mathrm{p}^{5}\left[{ }^{2} \mathrm{P}^{\circ}{ }_{3 / 2}\right] 4$ \\
\hline 6 & Ar I & $842.5 \mathrm{~nm}$ & $3 \mathrm{~s}^{2} 3 \mathrm{p}^{5}\left[{ }^{2} \mathrm{P}_{3 / 2}^{\circ}\right] 4 \mathrm{~s} \rightarrow 3 \mathrm{~s}^{2} 3 \mathrm{p}^{5}\left[{ }^{2} \mathrm{P}_{3 / 2}^{\circ}\right]$ \\
\hline 7 & Ar I & $852.1 \mathrm{~nm}$ & $3 \mathrm{~s}^{2} 3 \mathrm{p}^{5}\left[{ }^{2} \mathrm{P}_{1 / 2}^{\circ}\right] 4 \mathrm{~s} \rightarrow 3 \mathrm{~s}^{2} 3 \mathrm{p}^{5}\left[{ }^{2} \mathrm{P}^{\circ}{ }_{1 / 2}\right]$ \\
\hline 8 & Ar I & $912.3 \mathrm{~nm}$ & $3 \mathrm{~s}^{2} 3 \mathrm{p}^{5}\left[{ }^{2} \mathrm{P}^{\circ}{ }_{3 / 2}\right] 4 \mathrm{~s} \rightarrow 3 \mathrm{~s}^{2} 3 \mathrm{p}^{5}\left[{ }^{2} \mathrm{P}^{\circ}{ }_{3 / 2}\right] 4$ \\
\hline
\end{tabular}

The excitation temperature obtained from the emission intensity of the argon I lines were found to be in the range of $4477-7576 \mathrm{~K}$ with the pressure range of 0.1 to $2.0 \mathrm{MPa}$. The usage of copper as the electrode tip reveals that some luminescence in the copper spectra observed with the region between 327 and $578 \mathrm{~nm}$. The usage predominantly attached to $\mathrm{Cu} \mathrm{I}$ at the pressure of $0.1 \mathrm{MPa}$ while $\mathrm{Cu}$ II with the wavelength of $589.46 \mathrm{~nm}$ was detected at the entire pressure level (0.1 to 2.0MPa) as shown in Fig. 6 . 


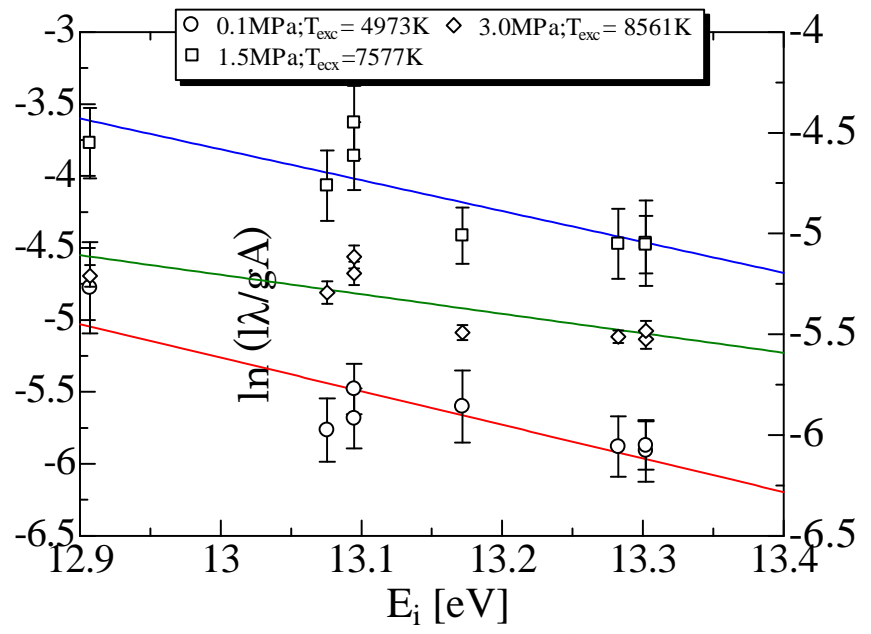

Fig.7 Boltzmann plots for measured intensity of argon I lines (flow rate $200 \mathrm{~mL} / \mathrm{min} \&$ pressure ranged $0.1-3.0 \mathrm{MPa}$ )

The excitation temperatures obtained at different gas pressures summarized in Fig. 8(a). It is revealed that the excitation temperatures increase with the increase of gas pressure. It can be referred to a higher frequency of electron collisions with the increase of pressure that affects to the excitation temperature enhancement [31]. The highest excitation temperature is obtained at 1.0MPa. However, any further increases in pressure consequence in raised excitation temperature.

Plasma irradiation performed along the argon flow rate range from 100 to $3000 \mathrm{~mL} / \mathrm{min}$, and it found that $T_{\text {exc }}$ comes to be reduced from 3960 to $2082 \mathrm{~K}$ with an increased flow rate of argon as shown in Fig. 8(b). The same trend reported by using micro-discharge at medium to high pressure in argon [34]. On the other hand, a decrease in excitation temperature with an increased pressure has been reported at low-pressure argon plasma discharge [25], low power microwave plasma [31], and high pressure of in-liquid plasma in water [30]. Fig. 8(b) shows that the excitation temperature tends to be decreased by an increase of argon flow rate. An identical trend reported at a high argon flow rate at atmospheric pressure for microwave plasma [26].

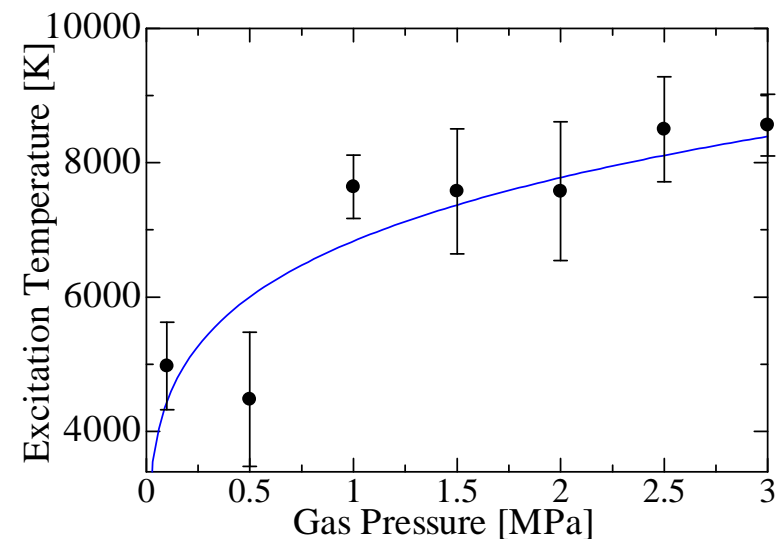

(a)

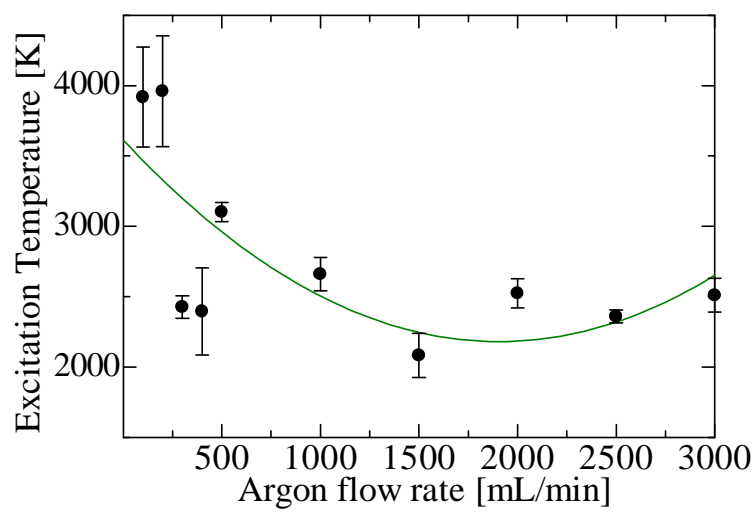

Fig. 8 Correlation of gas pressure (a) and argon flow rate (b) with excitation temperature of argon

\section{Influence of Electrode Materials and Plasma Impedance Stability}

The plasma irradiation performed by using two different materials for the tip of the electrode: tungsten and copper to investigate the effect of the type of electrode used on the argon plasma jet as shown in Fig.11(a) and 11(b). When the tungsten electrode used, the reflected power was unstable and difficult to adjust with the matching box. Conversely, it was much more stable using the copper electrode, and adjustment of the reflected power was fairly easy. However, melting and damage to the used of copper electrode was observed after the experiment.

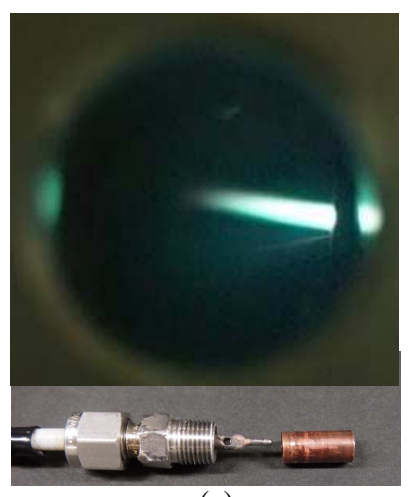

(a)

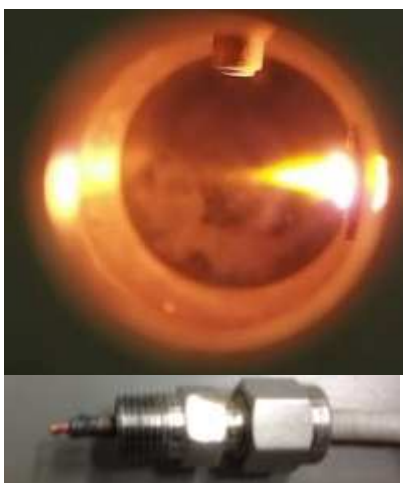

(b)
Fig.11 Effects of plasma jet from two types of electrode tip (a) Tungsten, (b) Copper

During the process of plasma irradiation, which maintained for about 60 seconds, the color of plasma turned out to be light yellow. Also, as the pressure increased between 0.1 and 2.0MPa, the reflection power was reduced. Consequently, the increased in-system pressure indicated an increase of net power supply [25].

\section{Methane Hydrate Decomposition by RF Argon Plasma Jet}

The gas yield from methane hydrate decomposition by the argon plasma jet concurs with the previous results [9], [10], for which hydrogen $\left(\mathrm{H}_{2}\right)$, carbon monoxide $(\mathrm{CO})$, carbon dioxide $\left(\mathrm{CO}_{2}\right)$, and methane $\left(\mathrm{CH}_{4}\right)$ were the main products. However, some hydrocarbon molecules such as $\mathrm{C}_{2} \mathrm{H}_{2}$ and $\mathrm{C}_{2} \mathrm{H}_{4}$ were not detected. The result of analysis of the gas yield from the hydrate plasma-induced decomposition is shown in Table 2 and on the bar chart in Fig.9. 
TABLE II

Result Of Analysis Of The Gas Produced From Hydrate DECOMPOSITION (NET INPUT POWER: 200w)

\begin{tabular}{|c|c|c|c|c|c|c|}
\hline \hline $\begin{array}{c}\text { Gas } \\
\text { Pressure } \\
(\mathbf{M p a})\end{array}$ & $\begin{array}{c}\text { Gas } \\
\text { production } \\
\text { rate }[\mathbf{m L} / \mathbf{s}]\end{array}$ & $\mathbf{H}_{\mathbf{2}} \%$ & $\mathbf{O}_{\mathbf{2}} \%$ & $\mathbf{C O \%}$ & $\begin{array}{c}\mathbf{C H}_{\mathbf{4}} \\
\mathbf{\%}\end{array}$ & $\begin{array}{c}\mathbf{C O}_{\mathbf{2}} \\
\mathbf{\%}\end{array}$ \\
\hline $\mathbf{0 . 1}$ & 1.67 & 27.69 & 11.20 & 2.26 & 56.30 & 2.55 \\
\hline $\mathbf{0 . 5}$ & 0.17 & 9.96 & 2.71 & 10.90 & 74.43 & 0.00 \\
\hline $\mathbf{1 . 0}$ & 0.10 & 4.97 & 2.57 & 3.76 & 88.70 & 0.00 \\
\hline $\mathbf{1 . 5}$ & 1.67 & 1.52 & 1.03 & 0.00 & 97.45 & 0.00 \\
\hline $\mathbf{2 . 0}$ & 0.67 & 2.33 & 9.52 & 0.00 & 94.81 & 0.00 \\
\hline
\end{tabular}

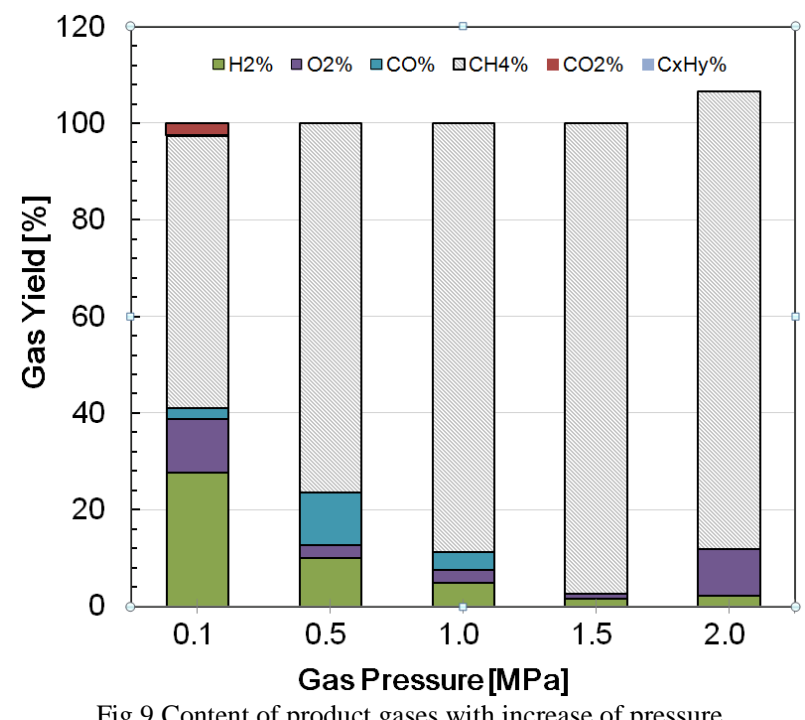

Despite argon plasma jet irradiation was successfully generated at high pressure, the hydrogen yield shows a tendency to decrease as the pressure increase. It is assumed that due to the simultaneous flow of argon gas during the process of plasma irradiation inside the reactor vessel, a substantial amount of $\mathrm{CH}_{4}$ was forced out earlier into the collecting bath of product gases before the decomposition process initiated. Consequently, the required basic reactions for the methane hydrate decomposition as shown in Eqs. (3) to (8) were not completely satisfied, due to an insignificant amount of $\mathrm{CH}_{4}$ that remained inside the reactor vessel. In the initial process, methane hydrate dissociation (MHD) reaction produced $\mathrm{CH}_{4}$ and $\mathrm{H}_{2} \mathrm{O}$ [9], [10]. Then the release of $\mathrm{CH}_{4}$ reacted with the water that turns into steam by the plasma simultaneously decomposed to yield $\mathrm{H}_{2}, \mathrm{CO}$, and $\mathrm{CO}_{2}$ by the reaction of steam methane reforming (SMR).

The required basic reactions for the decomposition process of methane hydrate are as follows:

$\diamond$ Methane hydrate dissociation (MHD):

1. $\mathrm{CH}_{4} \cdot 5 \mathrm{H}_{2} \mathrm{O} \rightarrow \mathrm{CH}_{4}+5 \mathrm{H}_{2} \mathrm{O}_{(\mathrm{g})}, \quad \boldsymbol{\Delta} \boldsymbol{H}=+53.5 \mathrm{~kJ} / \mathrm{mol} \mathrm{[35]}$

$\diamond$ Steam methane reforming (SMR) :

2. $\mathrm{CH}_{4}+\mathrm{H}_{2} \mathrm{O} \rightarrow 3 \mathrm{H}_{2}+\mathrm{CO}, \quad \boldsymbol{\Delta H}=+206.16 \mathrm{~kJ} / \mathrm{mol}$

3. $\mathrm{CO}+\mathrm{H}_{2} \mathrm{O} \rightarrow \mathrm{H}_{2}+\mathrm{CO}_{2}, \quad \boldsymbol{\Delta H}=-41.16 \mathrm{~kJ} / \mathrm{mol}$

$\diamond$ Methane cracking reaction (MCR) :

4. $\mathrm{CH}_{4} \rightarrow 2 \mathrm{H}_{2}+\mathrm{C}_{(\mathrm{s})}, \quad \boldsymbol{\Delta} \boldsymbol{H}=+74.87 \mathrm{~kJ} / \mathrm{mol}$

5. $2 \mathrm{CH}_{4} \rightarrow 3 \mathrm{H}_{2}+\mathrm{C}_{2} \mathrm{H}_{2}, \quad \boldsymbol{\Delta H}=376.47 \mathrm{~kJ} / \mathrm{mol}$

6. $2 \mathrm{CH}_{4} \rightarrow 2 \mathrm{H}_{2}+\mathrm{C}_{2} \mathrm{H}_{4}, \quad \boldsymbol{\Delta H}=202.21 \mathrm{~kJ} / \mathrm{mol}$

Notwithstanding that there is a trend for the rate of $\mathrm{H}_{2}$ production to decrease as the pressure increases by employing the argon plasma jet, the formation rate of carbon dioxide was observed to decrease (see Fig. 9). The hydrogen and $\mathrm{CO}$ production rates should vary simultaneously according to the methane steam methane reforming reaction. This suggests an alternative production mechanism for $\mathrm{CO}$, which is thought to be due to the pyrolysis of Teflon from the electrode. The absence of $\mathrm{C}_{2} \mathrm{H}_{2}$ and $\mathrm{C}_{2} \mathrm{H}_{4}$ as the byproducts observed from the content analysis of product gas by the gas chromatograph. It is to confirm that the methane-cracking reaction (MCR) only taken place to generated hydrogen and the $\mathrm{C}_{(\mathrm{s})}$, estimated by the balance of reaction in Eqs. (6).

The possibility of the hydrogen and the $\mathrm{C}_{(\mathrm{s})}$ generation as a by-product by methane-cracking reaction (MCR) also correspond to the Fig. 10 that illustrates the amount of effective energy (enthalpy) used for the decomposition of 1 mole of methane hydrate to produce hydrogen using the argon plasma jet depends on the pressure. Moreover, in a real hydrate system in a porous medium. $\mathrm{C}(\mathrm{s})$ attached to the reactor wall in the experiment also attach to the surface of the porous medium. The deposition of $\mathrm{C}_{(\mathrm{s})}$ on the porous medium could substantially reduce its permeability and let the blockage of the porous medium to interfere with hydrogen production [9].

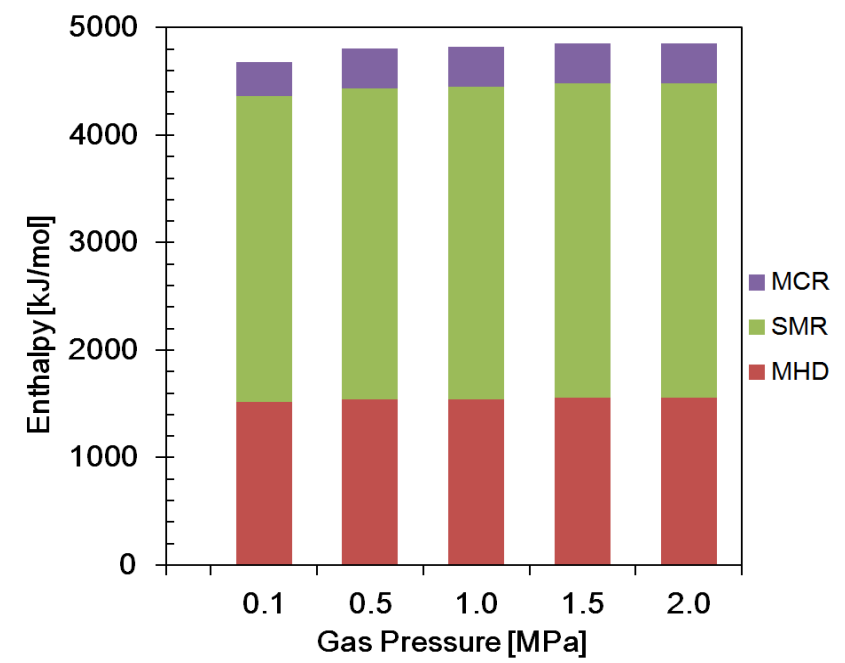

Fig.10 Net amount of energy used for methane hydrate decomposition by argon plasma Jet

Besides, Fig.10 also reveals that the enthalpies required during the process tend to remain constant as the pressure increases for the methane hydrate dissociation reaction (MHD), while the rest of primary reactions, i.e. Steam methane reforming (SMR) and methane cracking reaction (MCR) show a tendency to increase. In term of comparison with the other basic reactions, it was found that steam methane reforming (SMR) reaction became dominant concerning of converting methane into hydrogen.

\section{E. Efficiency of Hydrogen Production}

The hydrogen production efficiency determined by dividing the energy output in the outlet stream, defined as the molar flow of hydrogen multiplied by the lower heating value of hydrogen with the radio frequency input power for the plasma irradiation. This parameter should be considered as a significant factor regarding of performance of the argon 
plasma jet for methane hydrate decomposition process. This is not just a measurement of the methane hydrate decomposition process for hydrogen production but also an evaluation of energy efficiency that concerns to any future commercial cost.

The hydrogen production efficiencies depicted in Fig. 12. Although the hydrogen production efficiency is relatively low for argon plasma jet compared to RF in-liquid plasma method from the previous study, the reduction of carbon dioxide by the thermal decomposition of Teflon from carbon monoxide making it possible to be considered as an advanced promising technique. The hydrogen yield will become a significant challenge that must be improved in the future to enhance the hydrogen production efficiency of argon plasma jet.

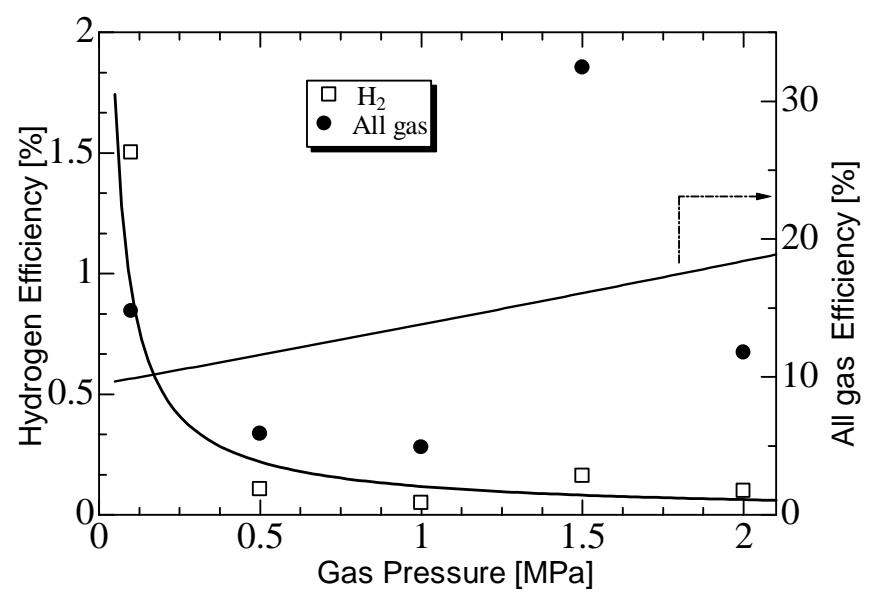

Fig.12 Efficiency of Hydrogen Production from decomposition of methane hydrate

In the previous study by Eka Putra et. al.[9], by the conventional in-liquid plasma method, plasma can only be generated at atmospheric pressure [9]. While using the argon plasma jet at the same input power of 200 Watt, the plasma can successfully to generate higher pressure than atmospheric pressure (range from $0.1 \mathrm{MPa}$ to $2.0 \mathrm{MPa}$ ). In practical condition, generating plasma under high pressure will give the result to the increase of input power. However, in the current study, by applying argon plasma jet, plasma can generate higher pressure with lower input power. Also, this study became the first time in observing the characteristic of argon plasma jet under very high pressure (2.0 MPa) mainly in the decomposition of methane hydrate.

\section{CONCLUSIONS}

Decomposition of methane hydrate has become feasible under high-pressure levels for hydrogen production. Even though the hydrogen production efficiency in the present study is less than that of the radio frequency plasma inliquid, the reduction of carbon dioxide by the thermal decomposition of Teflon from carbon monoxide making it possible to consider as an advanced promising technique in the future.

The excitation temperature has been determined from Boltzmann plot method with the pressure range of 0.1 to 2.0MPa. Due to a higher frequency of electron collisions from ions and atoms, the excitation temperature increases from 4477 - 7576K along with an increase of gas pressure, whereas along the argon flow rate range of 100 to $3000 \mathrm{~mL} / \mathrm{min}$, it reduces from 3960 to $2082 \mathrm{~K}$.

As the pressure increases, the enthalpy required during the process tend to remain constant for the methane hydrate dissociation reaction (MHD), while the rest of the first reaction, i.e., Steam Methane Reforming (SMR) and Methane Cracking Reaction (MCR) tend to increase. In comparison with the other necessary reactions, showed steam methane reforming (SMR) reaction become dominant concerning of methane conversion into hydrogen. The content analysis of product gas by the gas chromatography confirmed that Methane Cracking Reaction (MCR) taken place only to generated hydrogen and the $\mathrm{C}_{(\mathrm{s})}$ during plasma irradiation, due to the absence of $\mathrm{C}_{2} \mathrm{H}_{2}$ and $\mathrm{C}_{2} \mathrm{H}_{4}$ as the byproducts.

Despite the fact that the plasma irradiation generation is more stable at high pressures over that of the radio frequency plasma method, further improvement in the apparatus in the future is required to obtain higher hydrogen generation efficiency.

\section{REFERENCES}

[1] K. Siregar, "Strategy to reduce GHG emission and energy consumption at process production of biodiesel using catalyst from crude palm oil (CPO) and crude Jatropha curcas oil (CJCO) in Indonesia,” Int. J. Adv. Sci. Eng. Inf. Technol., vol. 5, no. 5, pp. 293299, 2015.

[2] Ayhan Demirbas, Methane Gas Hydrate. Springer, 2010.

[3] R. Rincón, a. Marinas, J. Muñoz, and M. D. Calzada, "Hydrogen production from ethanol decomposition by microwave plasma TIAGO torch,” Int. J. Hydrogen Energy, vol. 39, no. 22, pp. 1144111453, Jul. 2014.

[4] S. A. Kamal, J. M. Jahim, N. Anuar, O. Hassan, W. R. W. Daud, M. F. Mansor, and S. S. Rashid, "Pre-Treatment Effect of Palm Oil Mill Effluent (POME) during Hydrogen Production by a Local Isolate Clostridium butyricum,” Int. J. Adv. Sci. Eng. Inf. Technol., vol. 2, no. 4 , p. $325,2012$.

[5] E. Dendy Sloan and C. Koh, Clathrate Hydrates of Natural Gases, Third Edition, vol. 20074156. CRC Press, 2007.

[6] S. Nomura, A. E. E. Putra, S. Mukasa, H. Yamashita, and H. Toyota, "Plasma decomposition of clathrate hydrates by $2.45 \mathrm{GHz}$ microwave irradiation at atmospheric pressure," Appl. Phys. Express, vol. 4, no. 6, pp. 2-4, 2011.

[7] S. Nomura, H. Toyota, M. Tawara, H. Yamashita, and K. Matsumoto, "Fuel gas production by microwave plasma in liquid," Appl. Phys. Lett., vol. 88, no. 23, p. 231502, 2006.

[8] S. Nomura, H. Toyota, S. Mukasa, H. Yamashita, T. Maehara, and A Kawashima, "Production of hydrogen in a conventional microwave oven," J. Appl. Phys., vol. 106, no. 7, p. 73306, 2009.

[9] A. E. E. Putra, S. Nomura, S. Mukasa, and H. Toyota, "Hydrogen production by radio frequency plasma stimulation in methane hydrate at atmospheric pressure," Int. J. Hydrogen Energy, vol. 37, no. 21, pp. 16000-16005, Nov. 2012.

[10] I. Rahim, S. Nomura, S. Mukasa, and H. Toyota, "A Comparison of Methane Hydrate Decomposition Using Radio Frequency Plasma and Microwave Plasma Methods," in The 15th International Heat Transfer Conference, 2014, pp. 1-10.

[11] I. Rahim, S. Nomura, S. Mukasa, and H. Toyota, "Decomposition of methane hydrate for hydrogen production using microwave and radio frequency in-liquid plasma methods," Appl. Therm. Eng., vol. 90, 2015.

[12] X. Lu, M. Laroussi, and V. Puech, "On atmospheric-pressure nonequilibrium plasma jets and plasma bullets," Plasma Sources Sci. Technol., vol. 21, no. 3, p. 34005, 2012.

[13] S. I. Choi, J. S. Nam, C. M. Lee, S. S. Choi, J. I. Kim, J. M. Park, and S. H. Hong, "High purity synthesis of carbon nanotubes by methane decomposition using an arc-jet plasma," Curr. Appl. Phys., vol. 6, no. 2, pp. 224-229, Feb. 2006. 
[14] B. Vatzulik and I. Bica, "Production of magnetizable microparticles from metallurgic slag in argon plasma jet," J. Ind. Eng. Chem., vol. 15, pp. 423-429, 2009.

[15] I. Bica, "Iron micro-spheres generation in argon plasma jet," Mater. Sci. Eng. B Solid-State Mater. Adv. Technol., vol. 88, pp. 107-109, 2002.

[16] A. Sarani, N. De Geyter, A. Y. Nikiforov, R. Morent, C. Leys, J. Hubert, and F. Reniers, "Surface modification of PTFE using an atmospheric pressure plasma jet in argon and argon+CO2," Surf. Coatings Technol., vol. 206, no. 8-9, pp. 2226-2232, 2012.

[17] T. B. Huang, W. Z. Tang, F. X. Lu, J. Gracio, and N. Ali, "Argon-tohydrogen ratio in plasma jet diamond chemical vapour deposition," Surf. Coatings Technol., vol. 190, pp. 48-53, 2005.

[18] R. Burlica, K. Y. Shih, B. Hnatiuc, and B. R. Locke, "Hydrogen generation by pulsed gliding arc discharge plasma with sprays of alcohol solutions," Ind. Eng. Chem. Res., vol. 50, pp. 9466-9470, 2011.

[19] Y. C. Yang, B. J. Lee, and Y. N. Chun, "Characteristics of methane reforming using gliding arc reactor," Energy, vol. 34, pp. 172-177, 2009.

[20] M. G. Kong, B. N. Ganguly, and R. F. Hicks, "Plasma jets and plasma bullets," Plasma Sources Sci. Technol., vol. 21, no. 3, p. 30201, 2012.

[21] K. G. Kostov, M. Machida, and V. Prysiazhnyi, "Generation of Cold Argon Plasma Jet at the End of Flexible Plastic Tube," Plasma Sources Sci. Technol., vol. 24, 2012.

[22] A. Sarani, A. Y. Nikiforov, N. De Geyter, R. Morent, and C. Leys, "Characterization of an atmospheric pressure plasma jet and its application for treatment of non-woven textiles," Ispc_20, pp. 7-10, 2011.

[23] C. Giavarini and F. Maccioni, "Self-Preservation at Low Pressures of Methane Hydrates with Various Gas Contents," Ind. Eng. Chem. Res., vol. 43, no. 20, pp. 6616-6621, Sep. 2004.

[24] S. Circone, S. H. Kirby, and L. a Stern, "Direct measurement of methane hydrate composition along the hydrate equilibrium boundary.," J. Phys. Chem. B, vol. 109, no. 19, pp. 9468-75, May 2005.

[25] S. Nomura, S. Mukasa, H. Toyota, H. Miyake, H. Yamashita, T. Maehara, a Kawashima, and F. Abe, "Characteristics of in-liquid plasma in water under higher pressure than atmospheric pressure," Plasma Sources Sci. Technol., vol. 20, no. 3, p. 34012, Jun. 2011.
[26] Miotk, R.,Hrycak, B., Jasinski, M. and J. Mizeraczyk, "Spectroscopic study of atmospheric pressure $915 \mathrm{MHz}$ microwave plasma at high argon flow rate," J. Phys. Conf. Ser., vol. 406, p. 12033, 2012.

[27] Y. Hattori, S. Mukasa, H. Toyota, T. Inoue, and S. Nomura, "Continuous synthesis of magnesium-hydroxide, zinc-oxide, and silver nanoparticles by microwave plasma in water," Mater. Chem. Phys., vol. 131, no. 1-2, pp. 425-430, 2011.

[28] L. St-Onge, V. Detalle, and M. Sabsabi, "Enhanced laser-induced breakdown spectroscopy using the combination of fourth-harmonic and fundamental Nd:YAG laser pulses," Spectrochim. Acta Part B At. Spectrosc., vol. 57, pp. 121-135, 2002.

[29] N. M. Shaikh, S. Hafeez, and M. a. Baig, "Comparison of zinc and cadmium plasma parameters produced by laser-ablation," Spectrochim. Acta - Part B At. Spectrosc., vol. 62, pp. 1311-1320, 2007.

[30] H. Park and W. Choe, "Parametric study on excitation temperature and electron temperature in low pressure plasmas," Curr. Appl. Phys., vol. 10, pp. 1456-1460, 2010.

[31] F. Iza and J. a. Hopwood, "Rotational, vibrational, and excitation temperatures of a microwave-frequency microplasma," IEEE Trans. Plasma Sci., vol. 32, no. 2, pp. 498-504, 2004.

[32] B. Le Drogoff, J. Margot, M. Chaker, M. Sabsabi, O. Barthelemy, T W. Johnston, S. Laville, F. Vidal, and Y. von Kaenel, "Temporal characterization of femtosecond laser pulses induced plasma for spectrochemical analysis of aluminum alloys," Spectrochim. Acta Part B-Atomic Spectrosc., vol. 56, no. October 2000, pp. 987-1002, 2001.

[33] Physical Measurement Laboratory, "NIST: Atomic Spectra Database Lines Form," 2010. .

[34] B. N. Sismanoglu and C. L. a Cunha, "Optical and electrical diagnostics of microdischarges at moderate to high pressure in argon," vol. 40, no. 4, pp. 0-4, 2010.

[35] M. Mork and J. S. Gudmundsson, "Hydrate formation rate in a continuous stirred tank reactor: experimental results and bubble-tocrystal model," 4th Int. Conf. Gas Hydrates, May, pp. 19-23, 2002. 